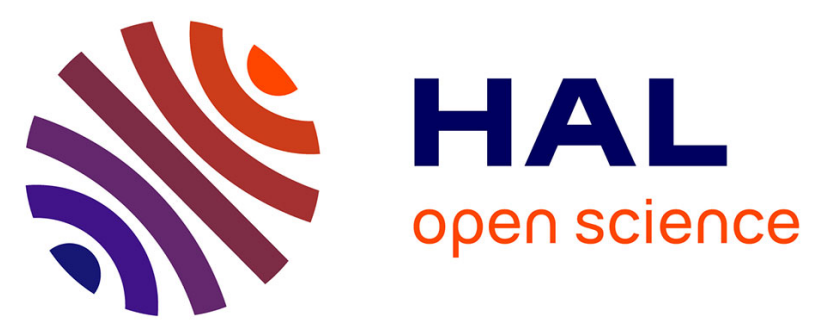

\title{
Le Petit Âge Glaciaire en milieu semi-aride: le bassin versant du Gialias (Chypre) et ses relations avec l'occupation des sols
}

\author{
Benoît Devillers, Nolwenn Lecuyer
}

\section{To cite this version:}

Benoît Devillers, Nolwenn Lecuyer. Le Petit Âge Glaciaire en milieu semi-aride : le bassin versant du Gialias (Chypre) et ses relations avec l'occupation des sols. Annales de Géomorphologie / Annals of Geomorphology / Zeitschrift für Geomorphologie, 2008, 50 (2), pp.207-224. halshs-00803770

\section{HAL Id: halshs-00803770 \\ https://shs.hal.science/halshs-00803770}

Submitted on 28 Mar 2013

HAL is a multi-disciplinary open access archive for the deposit and dissemination of scientific research documents, whether they are published or not. The documents may come from teaching and research institutions in France or abroad, or from public or private research centers.
L'archive ouverte pluridisciplinaire HAL, est destinée au dépôt et à la diffusion de documents scientifiques de niveau recherche, publiés ou non, émanant des établissements d'enseignement et de recherche français ou étrangers, des laboratoires publics ou privés. 


\begin{tabular}{|c|c|c|c|c|c|}
\hline Z. Geomorph. N. F. & 52 & 2 & $207-224$ & Berlin · Stuttgart & June 2008 \\
\hline
\end{tabular}

\title{
Le Petit Âge Glaciaire en milieu semi-aride : le bassin versant du Gialias (Chypre) et ses relations avec l'occupation des sols
}

\author{
par \\ Benoît Devillers, Valbonne et Nolwenn Lecuyer, Aix en Provence
}

avec 6 figures et 1 tableau

Résumé. Le Petit Âge Glaciaire (PAG) est décrit par divers auteurs comme une variation climatique fraîche et humide caractérisée par une augmentation de l'activité détritique et de la fréquence des crues au sein des hydrosystèmes de tout rang. Cette période coïncide d'autre part avec une importante exploitation agricole des sols. La conjonction de ces deux phénomènes a mené au constat de crise climato-anthropique des environnements durant cette période pour l'Europe occidentale. Cet article analyse le fonctionnement de l'hydrosystème du Gialias, dans l'île de Chypre, dans un contexte climatique de type méditerranéen semi-aride. La qualification et la quantification de la variabilité des dépôts de crue, par le croisement de la sédimentologie, la micropédologie et la minéralogie magnétique, sont datées par AMS ${ }^{14} \mathrm{C}$ et de nombreuses données archéologiques. Les changements environnementaux sont mis en relation avec l'occupation des sols, connue par les sources écrites et archéologiques. Les résultats montrent l'existence d'une forte accumulation entre le XIV et le XIX ${ }^{\mathrm{c}}$ siècle, formée par des crues de plaine de type "méditerranéen tempéré», plutôt qu'à des «crues éclair». Les rapports entre les variations hydro-sédimentaires et le peuplement justifient une redéfinition de la notion de crise environnementale durant le Petit Âge Glaciaire pour ce type de milieu.

Summary. The Little Ice Age (LIA) is known as a cool and humid climatic period characterized by an increase of detrital activity and flood frequency of all fluvial systems in the Mediterranean area (Pichard 1995, Greenbaum et al. 1999). In addition this period coincides with an increase in agricultural activity. The combination of these two phenomena has led several authors to describe a major climatic and anthropic crisis in southern France. The aim of this paper is to identify and quantify the dynamics of a semi-arid watershed during the Little Ice Age: the Gialias River, a major watershed in Cyprus. Changes in the nature and frequency of floods have been studied using sedimentology, micropedology and rock magnetism. The chronological background was obtained through $\mathrm{AMS}{ }^{14} \mathrm{C}$ and numerous archaeological dates. A clear link between fluvial changes and landuse variability was identified in the vicinity of the Potamia village. The results show a decrease in flash flood frequency during the LIA. High sedimentation rates can be explained by a greater occurrence of temperate floods, which yield less sediment than flash floods at the event scale. This combination of variations in stream flow and land use allow us to redefine the environmental crisis concept linked to the Little Ice Age for this type of dry mediterranean milieu. 


\section{Introduction, problématique}

L'étude de diverses sources historiques a mis en évidence une variation climatique fraîche et humide en Europe occidentale entre le milieu du XVI ${ }^{\mathrm{e}}$ et le milieu du XIX ${ }^{\mathrm{e}}$ siècle (Lamb 1977, 1979, Grove 1988, Le Roy Ladurie 1983). Ce «Petit Âge Glaciaire» (PAG) est en grande partie lié à l'Oscillation Nord Atlantique ou NAO (BoND et al. 1997), qui favoriserait une baisse des températures en Europe et une augmentation des précipitations (Lamp \& Peppler 1987, Gil et al. 2003). L'influence de la NAO est aussi sensible en Méditerranée orientale depuis plus de douze siècles, comme le prouvent la mesure des isotopes de l'oxygène ${ }^{16} \mathrm{O} /{ }^{18} \mathrm{O}$ en milieu marin en Israël (Schilman et al. 2001) ou celle des variations lacustres en Turquie (Jones et al. 2006). Toutefois le Proche Orient constitue une zone de transition entre l'influence des masses d'air atlantiques et la mousson indienne (Bolle 2003). Ainsi les variations climatiques enregistrées par la Mer Morte (ENZEL et al. 2003) et par les spéléothèmes israéliens (BAR-MATTHEWs et al. 1998) ne coïncident pas avec celles influencées par l'Oscillation Nord Atlantique. Le Petit Âge Glaciaire y est marqué par de faibles températures et une aridification progressive et régulière (Schilman et al. 2001).

En Europe occidentale et dans certaines régions du pourtour méditerranéen, le PAG correspond à des phases d'inondation et de détritisme (HUHMANN \& BRÜCKNER 2002, Zielhofer et al. 2002, MACKLin et al. 2002, 2006, FAUST et al. 2004, ThORndyCRAFT \& BENITO 2006). L'aggradation rapide dans les chenaux fluviaux conduit généralement à une «métamorphose» vers un style en tresses (BRAVARD 1989, SALVADOR et al. 1993, Huhmann et al. 2004). Cette évolution morphologique est aussi amplifiée par l'activité anthropique. Plusieurs auteurs ont mis en évidence une érosion accélérée en relation avec la mise en culture de nouveaux territoires et les défrichements qui gagnent progressivement les versants (Le Roy Ladurie 1983, Miramont et al. 1998, Bravard, 2002, Schult 2002, Marston et al. 2003, Lespez 2003).

Les travaux sur l'influence du Petit Âge Glaciaire en Méditerranée orientale sont beaucoup moins nombreux (Grove 1988). Certains mettent en évidence l'existence d'inondations entre les $\mathrm{XVI}^{\mathrm{e}}$ et XIX ${ }^{\mathrm{e}}$ siècles en Palestine, mais avec une fréquence très faible (LARonNe 1990, GreEnbaum et al. 1999). D'autres proposent un schéma d'évolution morphosédimentaire ou paléohydrologique parallèle à celui de Méditerranée occidentale (VITA-Finzi 1968, 1969, Cullen et al. 2002, Lespez, 2003, Issar 2003, KONDRASHOv sous presse). La position éloignée de Chypre par rapport aux influences de la NAO pose la question de l'intensité de la variation climatique du PAG pour ce pays et de la réponse morphogénique des bassin versants à cette histoire climatique régionale récente.

Actuellement les précipitations enregistrées à Chypre et sur la façade méditerranéenne du Moyen-Orient sont significativement corrélées avec celles de la Méditerranée occidentale. Mais, dans un contexte semi-aride, les hydrosystèmes y sont caractérisés par des écoulements sporadiques, voire violents, donnant lieu à des crues éclair particulièrement morphogènes (KARCz 1972, Devillers 2005).

L'objectif de ce travail est d'analyser les enregistrements sédimentaires récents du bassin-versant du Gialias (Mésorée), pour tenter de déterminer la nature des variations hydro-climatiques à Chypre au cours du PAG et de comprendre les interactions entre les dynamiques fluviales et le peuplement dans un contexte environnemental original, de type méditerranéen semi-aride. 
L'objet d'étude est le bassin versant du Gialias, en Messarée orientale, plaine centrale de l'île de Chypre (fig. 1A, B). Avec $340 \mathrm{~mm}$ de précipitations moyennes (NOAANCDC 2004), le bassin versant de $936 \mathrm{~km}^{2}$ appartient au domaine semi-aride (Grove 1977). Le Gialias est un fleuve de rang 7 (ordination de Strahler); la tête de bassin versant se situe dans le massif basaltique du Machairas à $1.450 \mathrm{~m}$ d'altitude; sa plaine alluviale, d'abord encaissée, s'élargit au contact des roches sédimentaires carbonatées du Tertiaire (grès, marnes, calcaires) entre Agia Varvara et Tymvou (fig. 1B). L'aval est constitué par une vaste ria holocène (Devillers et al. 2002). Les enregistrements sédimentaires étudiés se situent dans le secteur médian du bassin versant, la région de Potamia. Ce territoire a fait l'objet d'un projet de recherches pluridisciplinaires, sur la constitution des paysages médiévaux (dirigé par N. Lecuyer pour l'Ecole Française d'Athènes). Dans ce secteur, le Gialias et son affluent l'Alykos forment une plaine alluviale relativement large ( $2 \mathrm{~km}$ en moyenne) encadrée par des mesas formées de roches pliocènes ou pléistocènes (fig. 1B). Les plaines alluviales de l'Alykos et du Gialias comportent, selon les secteurs, une ou deux terrasses alluviales emboîtées, dominant les dépôts actuels de lit mineur. Elles correspondent à trois nappes alluviales (Devillers 2005), qui s'expriment ou non par des formes autonomes. A leur base, ces nappes reposent en discordance sur des formation pléistocènes $(P)$ ou le substratum pliocène $(\mathrm{PL})$. Les modalités et la chronologie des variations détritiques ont été étudiées par l'étude des faciès, de la sédimentologie, de l'épaisseur des séquences et de la minéralogie magnétique. L'histoire du peuplement et de la mise en valeur est perçue par les sources historiques et la collecte systématique de céramique.

Le site du moulin de Paleomylos a fait l'objet d'une fouille systématique qui permet de proposer un premier schéma chrono-stratigraphique. Il ne comporte qu'une seule terrasse, formée de trois nappes alluviales emboîtées: nappe A (épaisseur moyenne $12 \mathrm{~m})$, nappe $B(10 \mathrm{~m})$ et nappe $C(7 \mathrm{~m})$. Ces nappes sont séparées par des discordances de ravinement $(\delta 1, \delta 2$ et $\delta 3)$ de profondeurs variables. Les régressions linéaires obtenues grâce à 13 datations AMS ${ }^{14} \mathrm{C}$ et à 19 datations archéologiques permettent de dater respectivement la nappe A entre 11.990 et 4.430 B.P. cal., la nappe B entre 3.908 et 2.000 B. P. cal. et la nappe C entre 1.372 et 90 B. P. cal. (Devillers 2005). C'est la nappe alluviale C, correspondant à la période historique postérieure au XIV siècle, qui est étudiée ici. L'analyse repose sur la description de 14 profils sédimentaires, issus de coupes naturelles ou de fouilles archéologiques. Par ailleurs, de nombreux profils sériés ont été réalisés dans diverses configurations morphologiques sur les deux rives de l'Alykos et du Gialias, sur des tronçons rectilignes ou sur les rives concave et convexe des méandres libres du fleuve. Ils constituent un corpus de données qui autorise la restitution des dynamiques sédimentaires récentes et confirme les observations réalisées sur le site de Paleomylos. Le site de Paleomylos, puis les profils sériés des deux talwegs sont présentés dans les paragraphes suivants. 


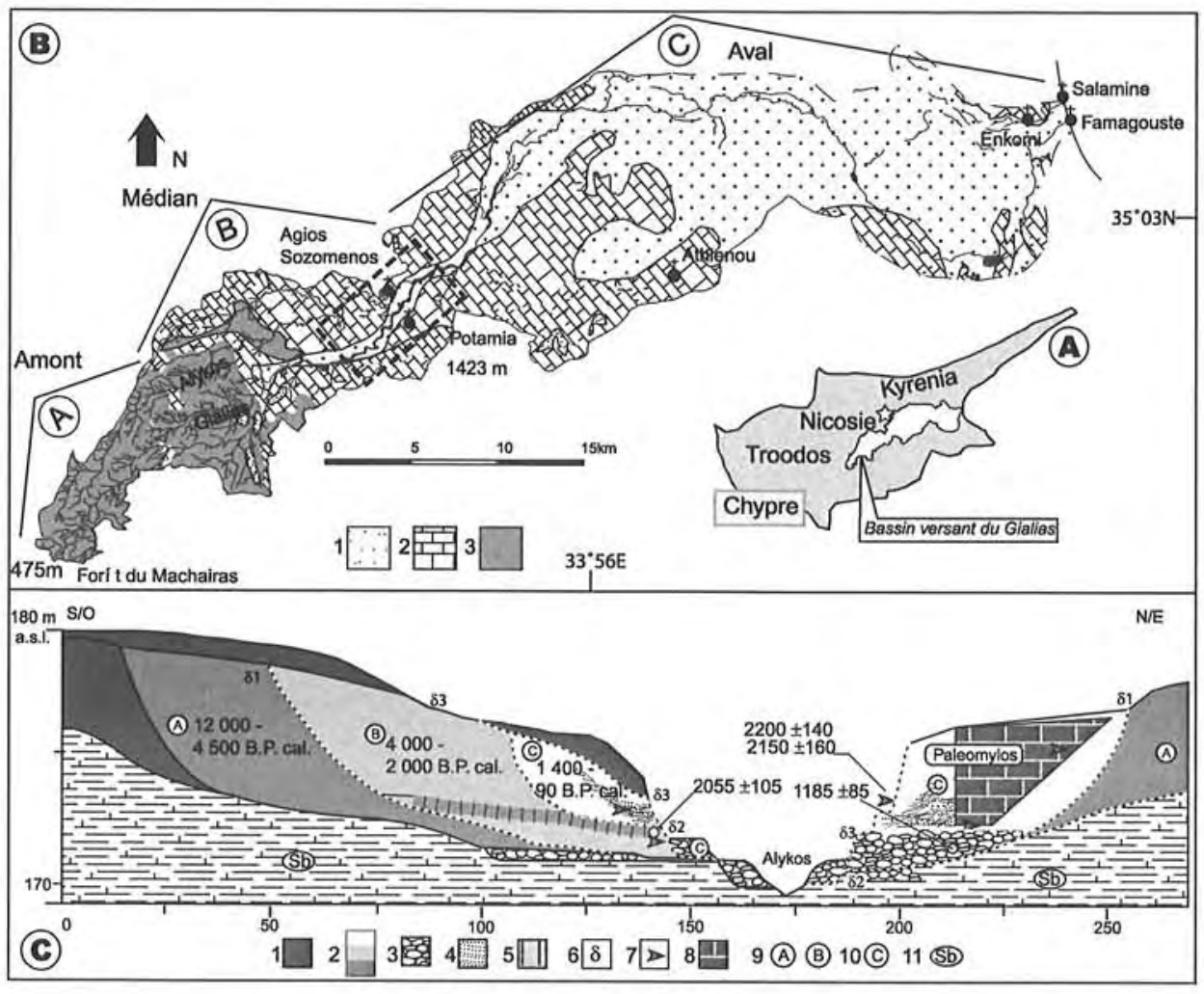

Fig. 1. Localisation, géologie et géomorphologie du bassin versant et des dépôts holocènes. A - Croquis de localisation. B - Le bassin versant du Gialias. 1 : alluvions et colluvions holocène ; 2 : roches sédimentaires, marno-calcaires, grès et poudingues, Paléogène à Pléistocène ; 3 : roches endogènes, Crétacé. $\mathrm{C}$ - Coupe en travers des dépôts holocènes près d'Agios Sozomenos. 1 : colluvions ; 2 : alluvions de lit majeur (plaine alluviale) ; $3:$ alluvions de lit mineur ; 4 : alluvions de lit moyen/berges ; 5 : paléosol, env. 2.000 ans B.P.; 6 : dépôts lacustres, Pléistocène récent $; 7$ : discordance de ravinement $; 8$ : datation archéologique ; 9 : moulin de Paleomylos ; 10 : formation alluviale ( $\mathrm{P}$ : Pléistocène) $; 11$ : terrasse à l'aval du profil.

Fig. 1. Location, watershed and Holocene alluvium geomorphology. A - Location sketch. B-The Gialias watershed. 1: Holocene deposits ; 2 : sedimentary rocks (marl, limestone, sandstone and conglomerate), Palaeogene to Pleistocene ; 3 : endogeneous rocks, Cretaceous. C - Cross section of Holocene deposits near Ayios Sozomenos. 1 : slope deposits ; 2 : floodplain deposits ; 3 : mainstream deposits ; 4 : bank deposits ; 5 : palaeosoil, ca. 2,000 yr B.P. ; 6 : lacustrine deposits, Late Pleistocene ; 7 : unconformity ; 8 : archaeological datation ; 9 : Paleomylos mill ; 10 : alluvial formation (P : Pleistocene) ; 11 : alluvial terrace downstream. 
Le moulin Franc de Paleomylos, appuyé sur le flanc de la nappe alluviale B, est presque totalement ennoyé dans les alluvions de la nappe alluviale $\mathrm{C}$ (fig. $1 \mathrm{C}$ et 2). Grâce aux repères chronologiques des niveaux archéologiques, ce site fournit un enregistrement très précis des dynamiques sédimentaires dans la moyenne vallée du Gialias. Le moulin était situé au bord du lit mineur pendant la durée de son fonctionnement, au cours de la période Franque (XIV e siècle). L'étude détaillée des stratigraphies et leur comparaison avec les stratigraphies des sites «naturels» montre que l'ouvrage n'influence pas sensiblement les taux de sédimentation et ne perturbe pas, ou peu, la dynamique de la sédimentation alluviale qui l'ennoie progressivement. Les fouilles ont affecté deux secteurs: la façade où est située la sortie des eaux, en relation directe avec le lit mineur de la rivière (secteur 2) et la salle des meules (secteur 1), plus en retrait, qui a servi de réceptacle aux inondations de l'Alykos (fig. 2 et 3).

\subsection{Stratigraphie et description des faciès}

Dans le secteur 2 (fig. 3 ), les premières fondations du moulin mises à jour (fig. 3 «Arc2002») sont creusées dans une nappe de galets homométriques (L: 8, 1:3 cm) sans matrice (unité 1). Cette nappe est datée de $1.185 \pm 85$ B.P. cal. (POZ-6617, tab. 1), soit

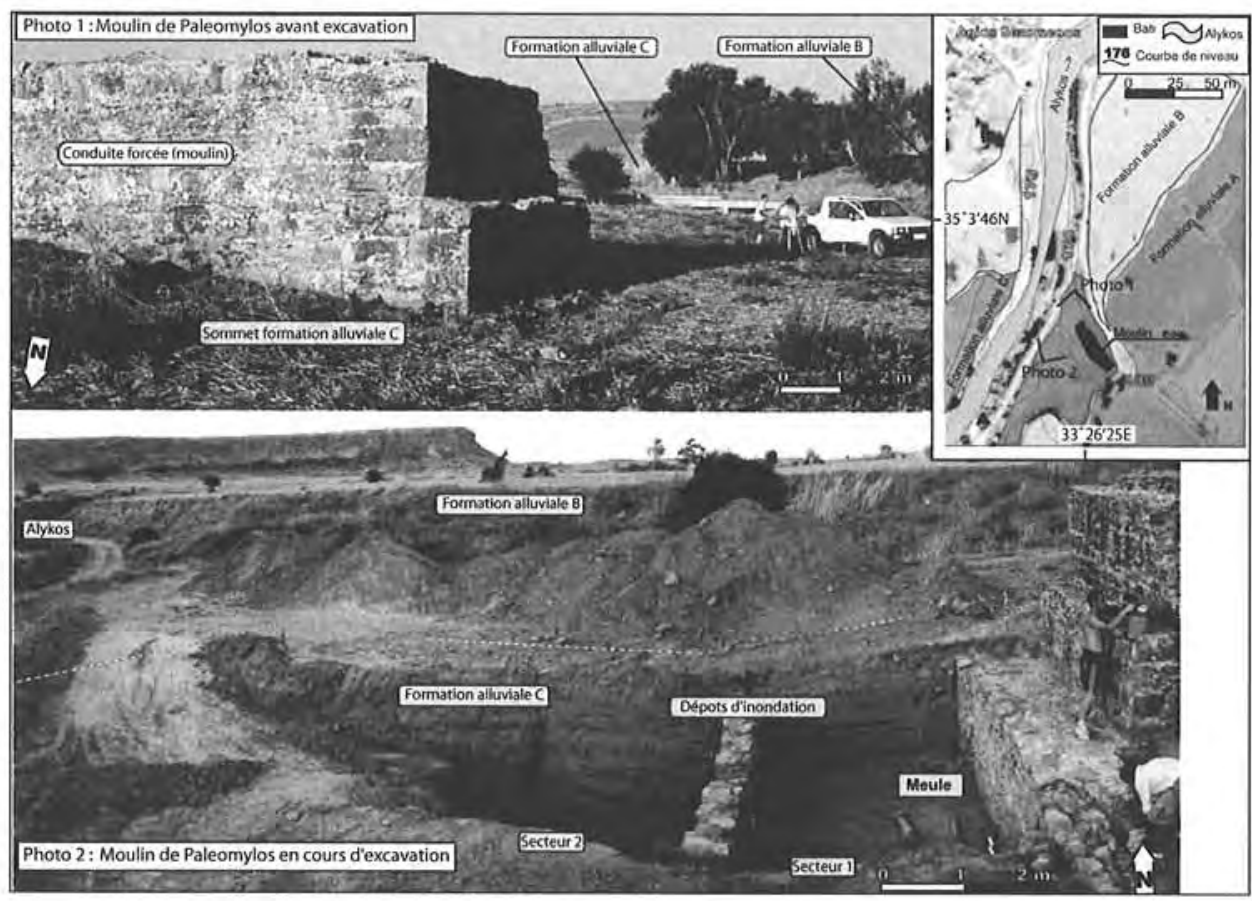

Fig. 2. Le site archéologique de Paleomylos.

Fig. 2. Paleomylos archaeological site. 
le $\mathrm{VIII}^{\mathrm{e}}$ siècle de notre ère, par des macro-restes végétaux recueillis à $7,5 \mathrm{~m}$ de profondeur (fig. 3). Les fondations du moulin, à $6,9 \mathrm{~m}$ de profondeur, puis la base des murs de renfort, jusqu'à $6,8 \mathrm{~m}$ ont été respectivement datées du XIV ${ }^{\mathrm{e}}$ et du XVI $\mathrm{XIè}^{\mathrm{e}}$ cle, grâce à l'étude des cortèges de céramiques (L. VALAURY, LAMM). Entre le VIII ${ }^{\mathrm{e}}$ et le XIV ${ }^{e}$, le lit mineur de l'Alykos s'exhausse donc d'environ 0,6 m. Le dernier mètre du dépôt caillouteux, qui recouvre la base des renforts, contient des céramiques du $\mathrm{XVI}^{\mathrm{e}}$ siècle, indiquant une accélération de l'aggradation du lit mineur (environ $1 \mathrm{~m}$ en 100 ans). À partir du sommet de la nappe caillouteuse, entre 0,5 et $5,5 \mathrm{~m}$ de profondeur, les alluvions changent de faciès et témoignent d'une forte accélération du taux de sédimentation (fig. 3 et $4 \mathrm{~A}$ ). Elles sont datées entre le $\mathrm{XVI}^{\mathrm{e}}$ siècle et la période ottomane grâce à la découverte d'une monnaie datée du XVIII ${ }^{e}$ siècle (1765) dans les

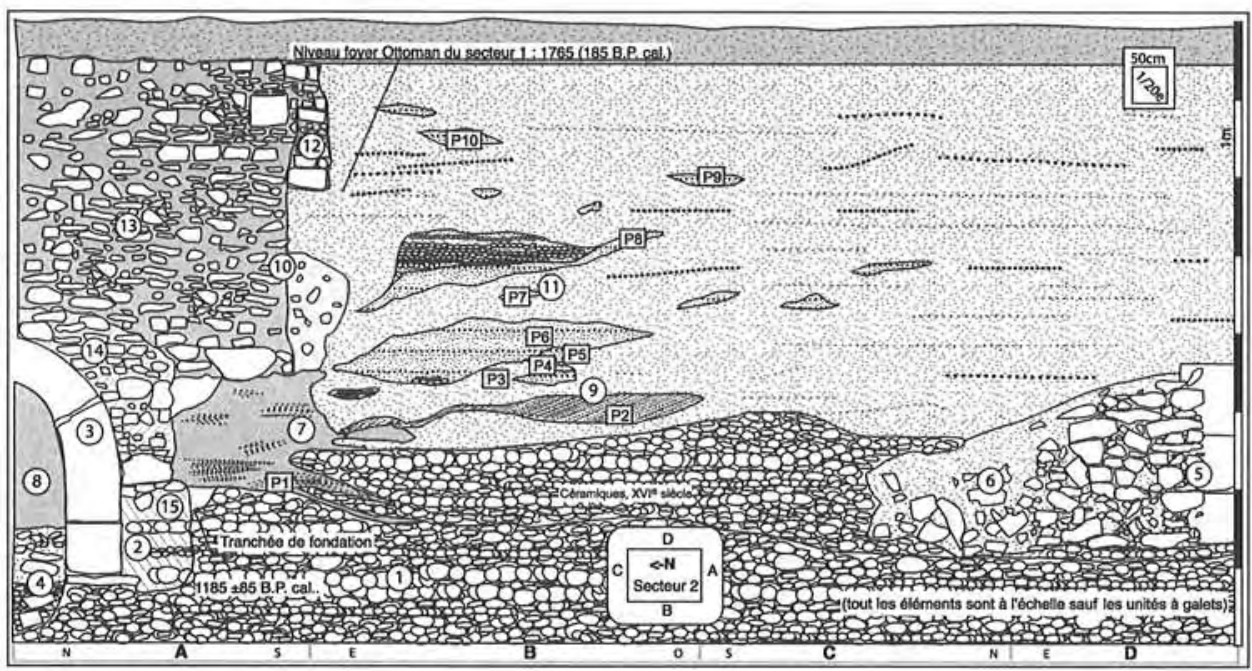

Fig. 3. Chronostratigraphie du secteur 2.1 : nappe graveleuse, lit mineur ; 2 : fondation du moulin ; 3 : arc de sortie des eaux, $1^{\text {ère }}$ phase de construction XIV ${ }^{\mathrm{c}}$ siècle ; 4 : curage et déblais de la sortie des eaux ; 5 : renfort, $2^{\mathrm{c}}$ phase de construction $\mathrm{XVI}^{\mathrm{e}}$ siècle $; 6$ : démantèlement du renfort ; 7 : alluvions (support de la $2^{\mathrm{e}}$ phase de construction; 8 : remblaiement de la salle des eaux (fin de l'utilisation du moulin) ; 9 : sables à litages obliques ; 10 : tranchée de fondation et mur d'adobe, $3^{\mathrm{e}}$ phase de construction; 11 : alluvions de lit majeur interstratifiées avec des lentilles de sables à litage oblique ; 12 : renfort de la partie supérieure du mur d'adobe, $4^{\mathrm{e}}$ phase de construction (185 ans B.P. cal. environ) ; 13 : mur d'adobe ; 14 : mortier (mur maçonné) ; 15 : chaux (consolidation des fondations).

Fig. 3. Sector 2 Chronostratigraphy. $1:$ pebble layer, mainstream $; 2$ : mill foundation; 3 : water discharge Arch, $1^{\text {st }}$ building phase XIV ${ }^{\text {th }}$ century $; 4$ : clearing out and cuts of the water discharge ; 5 : wall support, $2^{\text {nd }}$ building phase $\mathrm{XVI}^{\text {th }}$ century $; 6$ : dismantling of the support $; 7$ : Alluvium $\left(2^{\text {nd }}\right.$ building phase support $) ; 8$ : water room filling ; 9 : slanting bedded sands $; 10$ : sliced foundation and wall of adobe, $3^{\text {rd }}$ building phase ; 11 : overbank deposit interstratified with some slanting bedded sands ; 12 : higher part of the reinforcement of the adobe wall, $4^{\text {th }}$ phase of construction (Ca. 185 yr B.P. cal.); 13 : adobe wall ; 14 : mortar (built wall) ; $15:$ lime (consolidation of the foundations). 
mêmes faciès sur le secteur 1 (cf. infra). Elles sont caractérisées par des dépôts limonosableux, à micro-litage parallèle horizontal (faciès LP), avec parfois un grano-classement vertical positif (faciès GV). Dans leur partie inférieure s'intercalent des lentilles sablo-graveleuses, à micro-litage parallèle oblique (jusque vers $4,5 \mathrm{~m}$ ), puis à granoclassement vertical normal, enfin à petits galets et granules à litage horizontal vers $3 \mathrm{~m}$ de profondeur. La partie supérieure de la coupe est caractérisée par une augmentation sensible (de 50 à 100\%) de la taille des grains (médiane, 1 percentile) et du tri (Sorting index) (fig. 4A). Ce type d'évolution granulométrique traduit habituellement l'augmentation des débits liquides (Bravard \& Petit 1997, Arnaud-Fasseta $\&$ Provansal 2000). La susceptibilité magnétique massique a une variation relativement parallèle aux indices granulométriques, elle augmente à 4,5 mètres de la surface et atteint un maximum à 3,6 mètres de profondeur.

Dans le secteur 1 (salle des meules), les dépôts sont formés d'apports limono-sableux, sans lentilles. Des séquences à granoclassement vertical (faciès GV) s'y insèrent entre des lits horizontaux parallèles (faciès LP). Le sommet et la base de la stratigraphie sont constitués par des limons sableux homogènes, sans figure sédimentaire (faciès $\mathrm{SU}$ ). Les séquences de crues de type GV ou LP sont relativement moins épaisses ( $8 \mathrm{~mm}$ en moyenne) que celles de type $\mathrm{SU}$ ( $6 \mathrm{~cm}$ en moyenne). Toutefois leur forte fréquence souligne la persistance d'un régime hydrologique particulier à l'échelle pluri-centennale. Soixante huit séquences élémentaires d'épaisseur millimétrique à centimétrique y ont été individualisées et mesurées (fig. 4B). De nombreuses céramiques du XIV et du XVI ${ }^{\mathrm{e}}$ (L.Vallaury com. pers.) ont été collectées à la base de la coupe stratigraphique (fig. 4B). Trois artéfacts (monnaies et céramiques) permettent d'attribuer les séries situées à $2,5 \mathrm{~m}$ de profondeur à la période ottomane (XVIII ${ }^{\mathrm{e}}$ siècle.). A $1 \mathrm{~m}$ de la surface, les dépôts contiennent des céramiques et des outils du XIX ${ }^{\mathrm{e}}$ siècle.

\subsection{Interprétation bydro-sédimentaire et géomorphologique}

La nappe basale de galets a été mise en place par charriage de la charge de fond dans le chenal de lit mineur. On peut faire l'hypothèse que le tri et l'exportation des fines, après le dépôt des galets, correspondent à la persistance des écoulements au-delà des épisodes de crues (Osterkamp \& Costa 1987, Picard \& High 1973). Ce faciès s'oppose aux dépôts enrichis en fines, caractéristiques des lits mineurs intermittents des milieux semi-arides (Karcz 1972).

Les dépôts supérieurs, plus fins, correspondent aux dépôts des marges alluviales, berges et plaine d'inondation. Ces unités traduisent donc, d'une part le déplacement du chenal de lit mineur, d'autre part l'existence de débordements qui ennoient progressivement le moulin à partir du XVI ${ }^{\mathrm{e}}$ siècle. Les dépôts limono-sableux des secteurs 1 et 2 sont liés à des crues débordant hors du lit mineur. Les faciès limono-sableux à micro-litage parallèle horizontal (faciès LP), présentant parfois un grano-classement vertical positif (faciès GV), ont été déposés dans un contexte de suspension graduée, sous une couche d'eau assez profonde pour permettre une ségrégation verticale des différentes fractions granulométriques (VISHER 1969, WOODROFFE 2002). Ces dynamiques caractérisent le lit d'inondation proche, éventuellement même une levée alluviale de berge.

Les lentilles sablo-graveleuses résultent de courants plus dynamiques, chargés de sédiments grossiers en suspension graduée (PASsega 1957, Bravard 1986, SAlVA- 


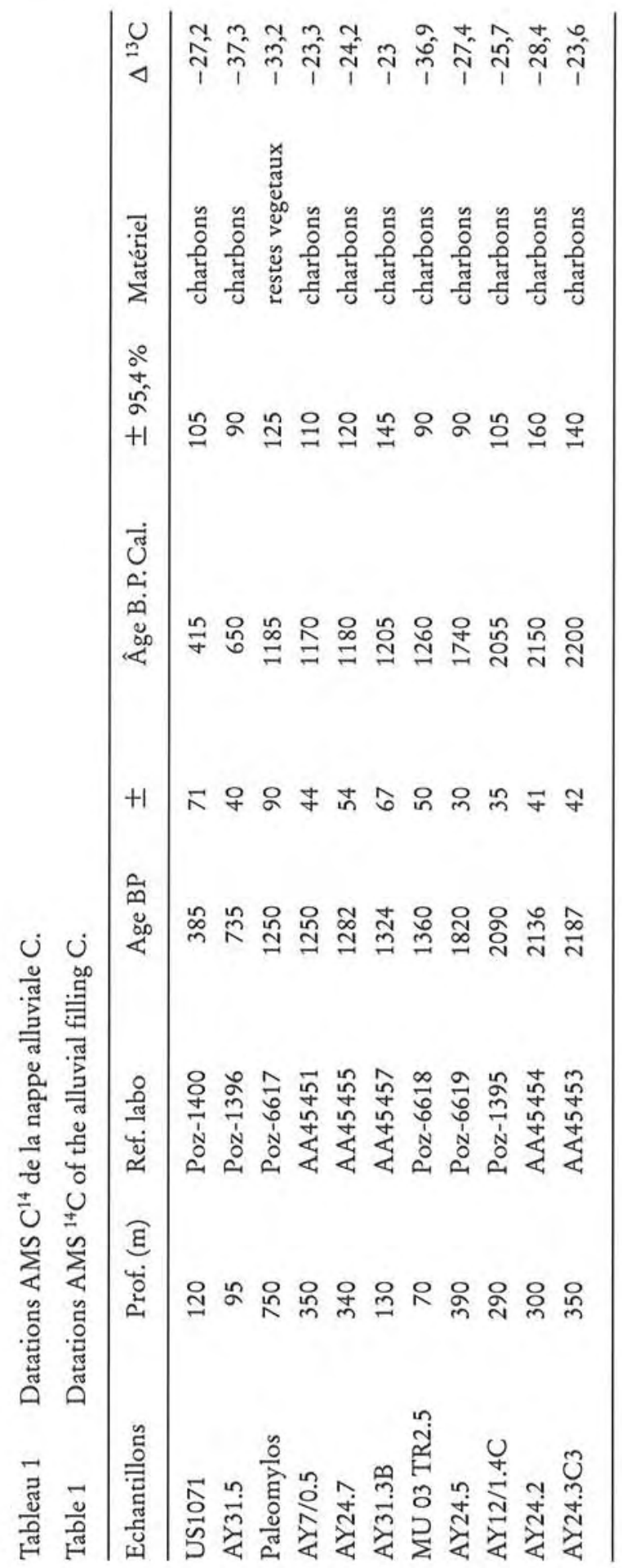




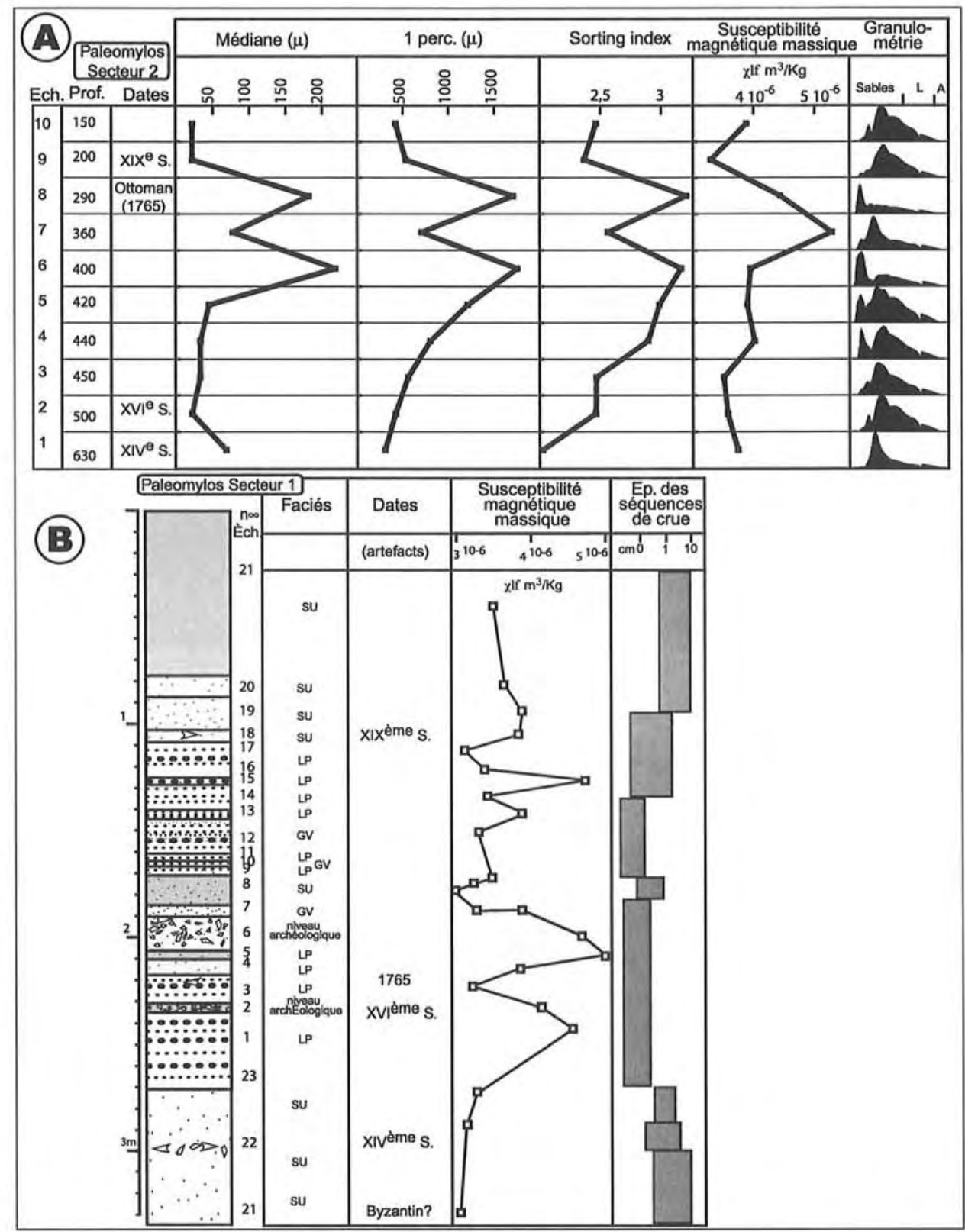

Fig. 4. Relevés stratigraphiques et sédimentologique du site archéologique de Paleomylos. A - Secteur II. B - Secteur I.

Fig. 4. Stratigraphics and sedimentological sketch of Palaeomylos site. A - Sector II. B - Sector I. 
DOR et al. 1993, Arnaud-Fassetta 1998, Visher 1969). En milieu fluvial, ce type de faciès peut caractériser les bancs dans le chenal, les berges ou les chenaux de crue (PiCARd \& High 1973). Les micro-litages parallèles obliques et les sables grano-classés évoquent les dépôts de bancs ou de berges. Mais la taille des lentilles, d'échelle métrique (de 1 à $3 \mathrm{~m}$ de large), et leur interstratification dans les dépôts plus fins montrent qu'elles correspondent à des «micro-chenaux» dans la zone d'inondation proximale. Ces faciès pourraient donc signaler une augmentation de la fréquence des crues débordantes et des débits solides, suffisamment importante à partir du XVI ${ }^{\mathrm{e}}$ siècle pour affecter la berge de l'Alykos, où était installé le moulin. Le grano-classement et le micro-litage bien organisé suggèrent une épaisseur d'eau et une durée relativement importantes de l'événement de crue.

Dans les dépôts de faciès uniforme (SU), le mélange d'une importante fraction fine (au moins $50 \%$ ) avec une faction sableuse (> à $100 \mu \mathrm{m}$ ) dont les grains sont non jointifs, pourrait être assimilé à un «dépôt en vrac», sans organisation interne, par des écoulements débordants, très chargés en matière en suspension et trop brefs pour évacuer les fines vers l'aval. Dans des contextes environnementaux similaires, ce type de faciès a été attribué par certains auteurs (Thomas 2000, Phillips 2002, Picard \& High 1973, Blair 2000) aux crues éclair des milieux arides à sub-arides (importance de la charge solide, faible durée de l'événement).

Sur le secteur 1, il est probable que la sédimentation ait été amplifiée dans la salle des meules, où l'eau entrante, brutalement freinée, avait des possibilités d'évacuations réduites (par deux portes sur les côtés nord et ouest). L'organisation stratigraphique et les faciès (à l'exception des lentilles, absentes ici) restent cependant similaires à ceux du secteur 2 et à celles des autres coupes stratigraphiques du Gialias (fig. $5 \mathrm{~A}$ et B), ce qui tend à minorer l'influence du bâtiment sur la circulation des eaux. On peut émettre l'hypothèse que, si la configuration des murs par rapport au dépôt a très influencé les faciès et les taux de sédimentation, les variations relatives de ceux-ci ne peut être qu'une conséquence des variations paléohydrologiques.

Les différents faciès de débordement sur la marge alluviale du Gialias autour du moulin permettent donc de distinguer deux modes de fonctionnement hydrologiques : le premier correspond à des crues débordant dans la plaine d'inondation proximale et dont les écoulements sont assez durables et dynamiques pour créer un microlitage visible et effectuer un tri granulométrique. Ce fonctionnement est comparable à celui de la plupart des organismes «méditerranéens» (Guilcher 1965). Le second est à rapprocher de celui des crues éclair des milieux semi-arides. Ce régime hydrologique semi-aride, mis en évidence par le changement de faciès des sables lités vers les dépôts de type SU, perdure jusqu'au XIX ${ }^{\mathrm{e}}$ Siècle.

Les mesures de susceptibilité magnétique permettent d'évaluer la capacité du transport du cours d'eau en mesurant la part relative des minéraux sources riches en fer (spinelles) provenant de la tête de bassin versant délivrant des magnétites et titanomagnétite de gros grains (env. $500 \chi^{\mathrm{lf}} \mathrm{A} / \mathrm{m}^{3} / \mathrm{kg}$ ) et celle des apports latéraux des roches sédimentaires carbonatées des secteurs médian et aval, aux valeurs très proches de zéro voire souvent négatives (Devillers 2005). Les autres minéraux pouvant intervenir dans le signal de susceptibilité comme la magnétite ultra fine d'origine pédologique sont négligeables dans le bassin versant (ThOMSON \& OLdField 1986, Deviller 2005). La susceptibilité magnétique massique est généralement importante : deux phases avec des maximums à environ $510^{-6} \chi^{1 \mathrm{f}} \mathrm{A} / \mathrm{m}^{3} / \mathrm{kg}$ apparaissent peu 
avant la période ottomane (1765) et la seconde avant le XIX ${ }^{\mathrm{e}}$ siècle (fig. 4). Ces données tendent à montrer que le transit longitudinal des sédiments était plus efficace durant le Petit Âge Glaciaire. En effet, les sédiments de la tête de bassin versant se retrouvent en plus grande proportion dans les dépôts alluviaux à l'aval durant cette période. Ce marqueur peut être aussi rapproché de la variation des différents faciès, qui tend à indiquer des écoulements plus longs et plus réguliers.

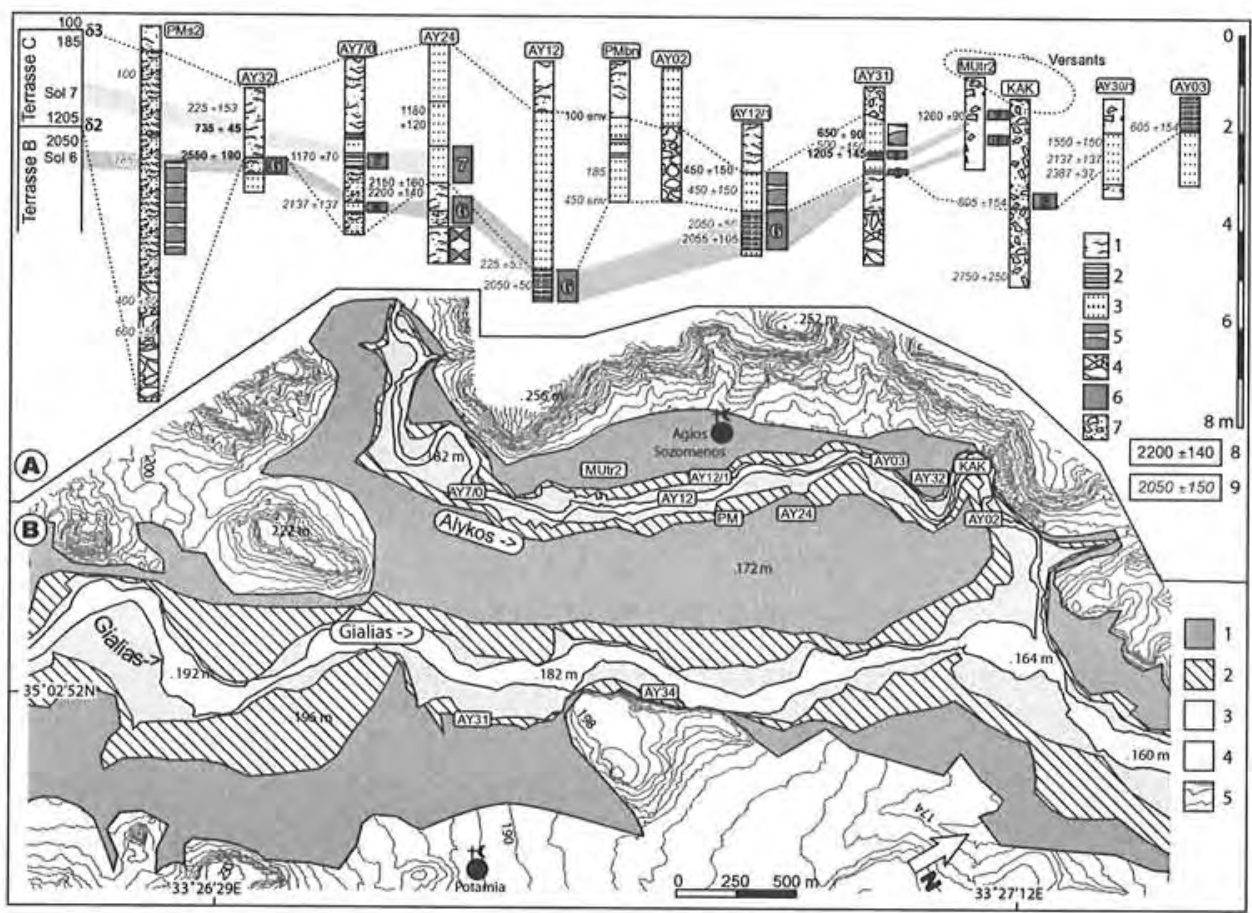

Fig. 5. Les dépôts alluviaux sur le territoire de Potamia. A - Chronostratigraphie des enregistrements sédimentaires de la terrasse alluviale C. 1 : faciès de suspension uniforme (SU), attribué aux crues éclair ; 2 : faciès à granoclassement vertical (GV) attribué aux crues méditerranéennes ; 3 : faciès intermédiaire à litage parallèle $(L P) ; 4$ : lentilles graveleuses; 5 : galets sans matrice, lit mineur ; $6:$ sol $; 7$ : colluvions ; $8:$ datation $\mathrm{AMS}{ }^{14} \mathrm{C} ; 9:$ datation archéologique. $\mathrm{B}$ - Cartographie géomorphologique des nappes alluviales holocènes (B). 1 : nappe alluviale $\mathrm{A}$ (11.200 à 5.100 ans B.P. cal.); 2 : nappe alluviale B (4.000 à 2.050 ans B.P. cal.); 3 : nappe alluviale $C$ (env. 1.205 à 100 ans B. P. cal.) 4 : plaine d'inondation actuelle; 5 : courbe de niveau (équidistance $2 \mathrm{~m}$ ).

Fig. 5. Alluvial deposit in Potamia village vicinity. A - Chronostratigraphy of the alluvial terrace C. 1 : uniform suspension facies (SU) assigned to flash floods; 2 : verticaly sorted facies (GV) assigned to Mediterranean type floods ; 3 : intermediate parallel bedding (LP) ; 4 : pebble intrusion ; 5 : openframework pebbles, mainstream ; $7:$ slope deposits ; 8: datation AMS ${ }^{14} \mathrm{C} ; 9$ : archaeological dating. B - Geomorphologic cartography of the Holocene alluvial fan. 1 : alluvial formation A $(11,200$ to $5,100 \mathrm{yr}$ B.P. cal. $) ; 2$ : alluvial formation B $(4,000-2,050 \mathrm{yr}$ B. P. cal.) ; 3 : alluvial formation C (ca. 1,205 to $100 \mathrm{yr}$ cal. B. P.) ; 4 : present floodplain ; 5 : contour line (equidistance $2 \mathrm{~m}$ ). 


\section{Impact morphologique et hydrologique du Petit Âge Glaciaire à l'échelle de la plaine du Gialias}

Bien que souvent moins complètes, d'autres archives sédimentaires permettent de confirmer et de préciser l'évolution hydro-morphologique mise en évidence lors des fouilles de Paleomylos. L'incision de la rivière postérieure à 1950, sur l'Alykos et le Gialias, a mis à jour la nappe alluviale $C$, au sein de laquelle 14 coupes stratigraphiques sériées ont été relevées (tab. 1, fig. 5A et B). L'épaisseur visible des dépôts dépend de leur position par rapport au lit actuel des cours d'eau et de la morphologie de la surface de ravinement $\delta 2$, dans laquelle la nappe C est emboîtée, qui détermine «l'es-

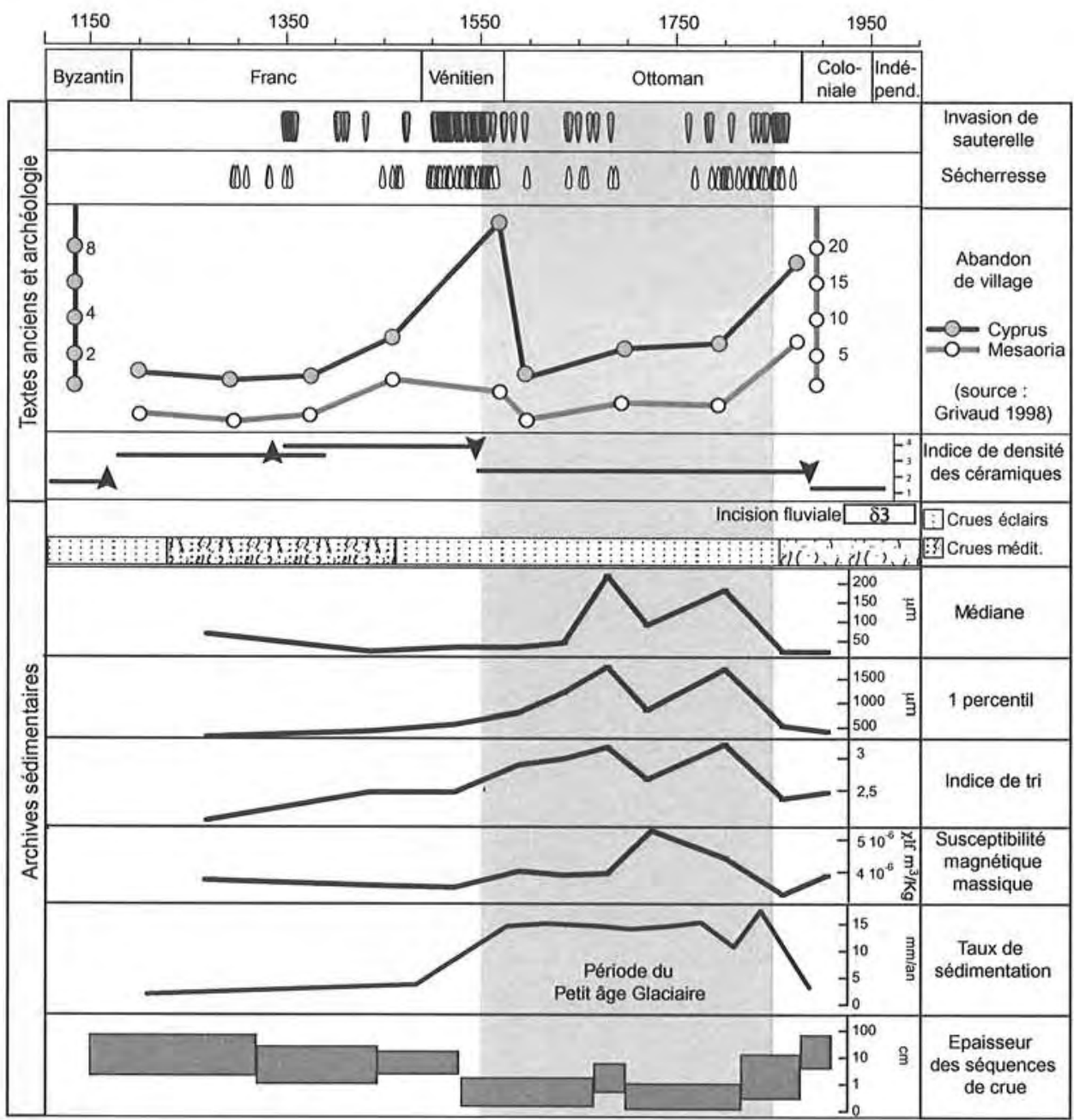

Fig.6. Évolution démographique, aléa climatique et rythme hydrologique.

Fig. 6. Demographic trends, climatic hazard and hydrological rhythm. 
pace de sédimentation» disponible. Trente et une datations AMS ${ }^{14} \mathrm{C}$ et archéologiques ont été réalisées. La date la plus ancienne (coupe AY32, fig. $5 \mathrm{~A}$ ) permet de dater le début de l'aggradation au milieu du $\mathrm{VIII}^{\mathrm{c}}$ siècle de notre ère. Le témoin le plus tardif est daté du XIX ${ }^{e}$ siècle (PMs2, PMbn, fig. 5A). L'aggradation de nappe alluviale C débuterait donc avant l'Optimum médiéval daté aux alentours du $\mathrm{XII}^{\mathrm{e}}$ siècle dans la région (Schilman et al. 2002). Cette période correspond en partie sur les coupes AY7/0, AY24, AY31, MUtr2, au le développement d'un paléosol embryonnaire brun calcique (fig. 5A). Certaines dates donnent toutefois un âge plus ancien pour ce sol (coupes AY31, Mutr2), qui s'est donc certainement développé sur une longue période. Toutefois l'essentiel de l'aggradation de la nappe alluvial C s'effectue après le XIV siècle (coupes Pms2n, AY12, Pmbn, AY02, AY31, Kak, AY03, fig. 5A) et relève donc pour l'essentiel du Petit Âge Glaciaire (fig. 5A), qui correspond donc à une dynamique d'exhaussement rapide de la plaine alluviale.

La base de la nappe, lorsqu'elle est visible, est souvent graveleuse, formée de galets en openwork. Son corps principal est limono-sableux, avec présence de lentilles graveleuses dans certaines coupes (AY12/1, AY 31, PMs2). Comme sur les fouilles de Paleomylos, on relève la présence de faciès de débordements à litage parallèle (LP) et à granoclassement normal (GV), caractéristiques de dépôts proximaux à une suspension graduée au sein de courants tractifs. La présence de lentilles graveleuses (coupe AY12, AY31, PM) pourrai correspondre à des paléo-chenaux de crue, indiquant une tendance au tressage. Cinq coupes, enfin, ont à leur sommet des faciès homogènes limono-argileux de type SU; toutefois l'érosion récente du sommet des nappes limite l'étude fine des dépôts les plus tardifs.

La nappe $\mathrm{C}$ enregistre donc une augmentation drastique des taux de sédimentation à partir du XVI ${ }^{\mathrm{e}}$ siècle (de 5 à $15 \mathrm{~mm} / \mathrm{an}$, fig. 6), qui témoigne d'une augmentation des débits solides. Les faciès, lités et granoclassés, révèlent des crues dont les hauteurs d'eau sont importantes, sans être des crues-éclair.

\section{Dynamique fluviale, crise environnementale et évolution du peuplement}

La figure 6 met en parallèle les différents marqueurs descriptifs des relevés sédimentaires avec les principaux indicateurs historiques. Bien que l'épaisseur des dépôts d'inondation, à l'échelle de la séquence de crue, diminue durant le PAG, les taux de sédimentation augmentent sensiblement durant cette période (fig. 6). Du point de vue morphogénique, l'augmentation de la fréquence des crues est donc plus importante que la diminution des volumes déposés lors d'une inondation. Ces mutations sont induites par le changement de la nature des crues (crues à caractère tempéré). Le Petit Âge Glaciaire est donc, à l'instar de ce qui est observé sur les rives Nord de la Méditerranée, particulièrement morphogène dans le contexte semi-aride de Chypre. La dernière incision $\delta 3$ est très récente puisqu'elle est postérieure à différents barrages submersibles construits entre 1952 et 1960 . La consommation en eau et l'établissement de réservoirs importants ont certainement amplifié les effets de la diminution moyenne des précipitations (Issar 2003, NOAA-NCDC 2004).

La comparaison des résultats obtenus par les archives sédimentaires, par les sources écrites et les données archéologiques (fig. 6) permet de définir la nature des impacts des changements environnementaux sur l'occupation des sols. Le recensement de l'abandon des villages, des années sèches et des invasions de sauterelles dans 
les archives franques, vénitiennes et ottomanes à Chypre est réalisé par Grivaud (1998). L'indice de densité de céramiques est fondé sur la collecte systématique (transect de $5 \mathrm{~m}$ ) des tessons sur l'ensemble du territoire de Potamia effectuée de 1999 à 2004 (Dir. N. Lecuyer pour l'EFA). L'indice représente le nombre total de tessons identifiés (L. VALLAury \& V. FrançOIS, LAMM) rapporté au nombre d'années de la période de production dont ils sont issus. Cet indice est donc un marqueur de la densité de l'occupation des sols.

Les données montrent un fort synchronisme entre les doléances émises par les Chypriotes en raison des sécheresses et celles consécutives aux invasions de sauterelles qui ruinaient les cultures. Ce phénomène est particulièrement marqué à l'époque vénitienne et à la fin de l'époque ottomane (fig. 6); il est en revanche très peu présent durant le Petit Âge Glaciaire. La sécheresse s'exprime par des précipitations moindres, par une plus forte intermittence fluviale (crues éclair) et par une raréfaction des écoulements. Elle est donc particulièrement redoutée pour les cultures pluviales et irriguées (céréales et coton, CHRISTODOULOU, 1959) et peut mener à terme à la famine en Messarée (Grivaud 1998). Les indices d'occupation des sols (abandon de village, GRIVAUD 1998 et concentration en tessons, Survey Potamia, N. Lecuyer Dir.) montrent un recul marqué de l'occupation des sols durant les périodes postérieure et surtout antérieure au Petit Âge Glaciaire (fig. 6).

Les conditions hydrologiques du Petit Âge Glaciaire semblent moins contraignantes, l'importance et la relative régularité des flux hydriques plus importants et plus fréquents, entraînent des conditions favorables à la culture irriguée et permettent l'exportation de surplus de Chypre vers l'Europe occidentale (CHristodoulou 1959). Les calamités comme les invasions de sauterelles se font aussi plus rares. Parallèlement, on note la stabilisation des implantations villageoises et de l'occupation des sols (fig. 6). Les effets du Petit Âge Glaciaire souvent perçus de façon négative en Méditerranée occidentale sont ici atténués par le contexte bioclimatique semi-aride des vastes plaines intérieures chypriotes où la contrainte principale des activités agricoles est l'accès à l'eau pour les cultures pluviales et irriguées. L'augmentation des taux de sédimentation a toutefois un impact sur les aménagements hydrauliques comme le moulin de Paleomylos, d'autres exemples de ce type sont nombreux à Chypre (moulins d'Archangelos, d'Akaki, ponts Francs et Vénitiens de Messarée orientale, etc.). Cette accélération de la sédimentation semble être le résultat d'une érosion accélérée sur les versants, comme semblent le montrer les fortes accumulations colluviales de cette époque relevées sur les coupes Kak et Mu (fig. 5A, Devillers 2005). Des réponses techniques dans ce milieu rural sont utilisées (aménagement en terrasses, aménagement de berges), mais leur efficacité réelle à l'échelle micro-régionale semble être limitée.

\section{Conclusion}

Les archives sédimentaires du bassin versant du Gialias mettent en évidence l'influence morphologique du Petit Âge Glaciaire en Méditerranée orientale. L'évolution hydro-sédimentaire du système fluvial est parallèle à celle décrite en Europe du Sud (Bravard 1989, Arnaud-Fasseta \& Provansal 1999, Gutierrez-Elorza \& PenaMONNE 1998, MaCKLin et al. 2006). Elle se manifeste principalement par l'augmentation des taux de sédimentation moyens malgré une diminution de l'épaisseur des dépôts à l'échelle de la séquence de crue et par une tendance au tressage. Cependant, 
le milieu semi-aride de Chypre entraîne des nuances quant à l'évolution des milieux fluviaux durant cette période, qui est marquée par l'augmentation des fréquences d'inondation, de la régularité des flux et par la diminution des crues éclair.

Bien qu'encore sporadiques, les différentes données pluridisciplinaires mettent en évidence les modalités de l'influence des dynamiques environnementales sur l'occupation des sols (Bousquet \& Pechoux 1980). Les effets négatifs du Petit Âge Glaciaire sur les sols (érosion, inondation, perte des récoltes, etc.) (BERGER 1998, LE Roy Ladurie 1983, Jorda \& Provansal 1996, Neboit-Guilhot 1999, Xoplaki et al. 2001, Devillers \& Provansal 2003) semblent secondaires par rapport à l'aléa hydrologique des périodes de sécheresse durant la domination vénitienne et à la fin de la période ottomane. La notion de crise des environnements développée en Méditerranée occidentale, caractérisée par une péjoration climatique fraîche et humide, ne peut donc pas s'appliquer au Petit Âge Glaciaire des milieux semi-arides de Méditerranée orientale. A l'échelle macro-régionale, on observe donc un renversement des périodes à forte contrainte environnementale vis-à-vis de l'occupation des sols, principalement en raison des conditions d'accès aux ressources en eau.

\section{Remerciements}

Les auteurs remercient l'Ecole Française d'Athènes, G.Petrides et I.Panayides (Geological Survey of Cyprus), L.Purdue (CEPAM), P. Rochette (CEREGE), B.Simon (CEREGE), L. Valaury et V. François (LAMM) pour leur aide logistique et scientifique. Les auteurs tiennent aussi à remercier J.-P.Bravard, M.Fort, C.Morhange, M. Provansal, et bien sur, J.-C. Thouret pour leurs judicieux commentaires.

\section{Références}

Arnaud-Fasseta, G. \& Provansal, M. (1999): High frequency variations of water flux and sediment discharge during the Little Ice Age (1586-1725 AD) in the Rhône Delta (Mediterranean France). Relationship to the catchment basin. - Hydrobiologia 410: 241-250.

Bar-Matthews, M., Ayalon, M. \& Kaufmann, A. (1998): Middle to Late Holocene (6.500 yr period) Paleoclimate in the Eastern Mediterranean region from stable isotopic composition of speleothems from Soreq Cave, Israel. - In: Issar, A. \& Brown, A. (eds.): Water, Environment and Society in Times of Climatic Change: 203-215; Kluwer Academic Publishers, Dordrecht.

BERGER, J.-F. (1996): Le cadre paléogéographique des occupations du bassin valdanais (Drôme) à l'Holocène. - Thèse, université de Paris I, Paris, 320pp.

BLAIR, T. C. (2000): Sedimentology and progressive tectonic unconformities of the sheetflooddominated Hell's Gate alluvial fan, Death Valley, California. - Sedimentary Geol. 132: 233-262.

Bolle, H.-J. (2003): Mediterranean Climate: Variability and Trends. - In: Regional Climate studies. 320 pp. Springer, Munich.

Bond, G., Showers, W., Cheseby, M., Lotti, R., Almasi, P., De Menocal, P., Priore, P., Cullen, H., Hadjas, I. \& Bonani, G. (1997): A pervasive millenial-scale cycle in North Atlantic Holocene and glacial climates. - Science 278: 1257-1266.

Bourke, A. (1984): Impact of the Climatic fluctuation on European Agriculture. - In: FloHN, H. \& Fantechi, R. (eds.): The Climate of Europe: Past, Present and Future: 269-313; Reideil Publ. Company, Lancaster. 
Bousquet, B. \& Pechoux, P.-Y. (1980): Géomorphologie, archéologie, histoire dans le bassin orientale de la Méditerranée: principes, méthodes résultats préliminaires. - Méditerranée 1: 33-45.

BRAvard, J.P. (1989): La métamorphose des rivières des Alpes françaises à la fin du MoyenAge et à l'époque moderne. - Bull. de la Soc. Géographique de Liège 145-157: 25.

- (2002): Le «traitement» des versants dans le département de la Drôme. Des inondations de 1840 à la loi du 27 Juillet 1860. - Ann. des Ponts et Chaussées 103: 37-43.

Bravard, J.-P. \& Petrt, F. (1997): Les cours d'eau. Dynamique du système fluvial. - Armand Colin, Paris, 222pp.

Brewer, S., Alleaume, S. \& Guiot, J. (2006): Historical droughts in Mediterranean regions during the last 500 years: a data/model approach. - In: Ganssen, G., Clauussen, M., Rousseau, D.-D. \& Wolff, E. (eds.): Climate of the Past 11; 11 pp.

Christodoulou, D. (1959): The evolution of the rural land use pattern in Cyprus. - Cornwall, $230 \mathrm{pp}$.

Cullen, H. M., Kaplan, A., Arkin, P. A. \& De Menocal, P.B. (2002): Impact of the North Atlantic Oscillation on middle eastern climate and streamflow. - Climate Change 55: 315-338.

Devillers, B. (2003): Holocene morphogenesis and land use in eastern Mesaoria (Cyprus), preliminary results. - In: FouACHE, E. (ed.): Dynamiques environnementales et Histoire en milieux méditerranéens: 361-369; Elsevier.

- (2005): Morphogenèse et anthropisation holocènes d'un bassin versant semi-aride: le Gialias, Chypre. - Thèse de doctorat, université de Provence, Aix en Provence, 420pp.

Devillers, B., Morhange, C., Buffière de l'Air, M., Bourcier, M. \& Provansal, M. (2002): Détritisme, potentialités et aménagements du territoire à l'Age du Bronze sur les secteurs amont (Potamia-Agios Sozomenos) et aval Messarée orientale, secteur d'Enkomi-Acheritou) du bassin versant du Gialias (Chypre). - Cahier du Centre d'Étude Chypriotes 32: 33-52.

Devillers, B. \& Provansal, M. (2003): La morphogenèse d'un géosystème cultivé depuis le Néolithique Récent: les petits bassins versants de la moyenne vallée de l'Hérault, - Géomorphologie, Relief, Processus, Environnement 2: 83-98.

Enzel, Y., Bookman, R., Sharon, D., Gvirtzman, H., Davan, U., Ziv, B. \& Stein, M. (2003): Late Holocene climates of the Near East deduced from Dead Sea level variations and modern regional winter rainfall. - Quatern. Res. 60: 263-273.

Faust, C.Z., Olmo, F. D. D. \& Escudero, R. B. (2002): Sedimentation and soil formation phases in the Ghardimaou Basin (northern Tunisia) during the Holocene. - Quatern. Internat. 93: 109-125.

Faust, D., Zielhofer, C., Escudero, R. B. \& Olmo, F. D. D. (2004): High-resolution fluvial record of late Holocene geomorphic change in northern Tunisia: climatic or human impact. - Quatern. Science Reviews 23: 1757-1775.

Gil, I.M., Abrantes, F. \& Hebbeln, D. (2006): The North Atlantic Oscillation forcing through the last 2000 years: Spatial variability revealed by high-resolution marine diatom records from N and SW Europe. - Marine Micropaleontol. 603: 113-129.

Gob, F., Petit, F., Bravard, J.-P., Ozer, A. \& Gob, A. (2003): Lichenometric application to historical and subrecent dynamics and sediment transport of a Corsican stream (Figarella River - France) . - Quatern. Science Reviews 22: 2111-2124.

Greenbaum, N., Schick, A.P. \& Baker, V.R. (2000): The palaeoflood record of a hyperarid catchment, Nahal Zin, Negev desert, Israel. - Earth Surf., Proc. and Landf. 25: 951-971.

Grivaud, G. (1998). - Villages désertés à Chypre (fin XII - Fin XIX ${ }^{\mathrm{c}}$ siècle). - MELATAI KAI YPOMNHMATA III, Nicosie, 536pp.

Grove, A. T. (1977): The geography of semi-arid lands. - In: Philosophical Transaction of the Royal Soc. London, Series B, 278: 457-475. 
Grove, J. M. (1988): The Little Ice Age. - Methuen \& Co, Cambridge, 498 pp.

Gutierrez-Elorza, M. \& Pena-Monne, J. L. (1998): Geomorphology and late Holocene climatic change in Northeastern Spain. - Geomorphology 23: 205-217.

Guilcher, A. (1965): Précis d'Hydrologie. - Masson, Paris, 389 pp.

Huhmann, M. \& BrücKNeR, H. (2002): Holocene terraces of the upper Dnister. Fluvialmorphodynamics as a relation to climate changes and human impact. - Z. Geomorph. N.F. 127: $67-80$.

Huhmann, M., Kremenetski, K. V., Hiller, A. \& Brückner, H. (2004): Late quaternary landscape evolution of the upper Dnister valley, western Ukraine. - Palaeogeogr., Palaeoclimatol., Palaeoecol. 209: 51-71.

Issar, A. S. (2003): Climate changes during the Holocene and their impact on hydrological systems. International Hydrology series. - Cambridge Univ. Press, Cambridge, $124 \mathrm{pp}$.

Jones, M. D., Roberts, C. N., Leng, M. J. \& Türkes, M. (2006): A high resolution Late Holocene lake Isotope record from Turkey and links to North Atlantic and monsoon climate. - Geology 34, 5: 361-364.

Jorda, M. \& Provansal, M. (1996): Impact de l'anthropisation et du climat sur le détritisme dans le Sud-Est de la France (Alpes du Sud et Provence). - Bull. de la Soc. Géol. de France 167: 159-168.

KARCZ, I. (1972): Sedimentary structures formed by flash floods in southern Israel. - Sediment. Geol. 7: 161-182.

Kondrashov, D., Feliks, Y. \& Ghil, M. (Sous presse): Oscillatory modes of extended Nile River records (A. D. 622-1922). - Geophys. Res. Letters 10: 4.

LAмв, H.H. (1977): Climate, History and the Modern World. - Methuen, London, $835 \mathrm{pp}$.

- (1979): Climatic variation and changes in the wind and ocean circulation: The Little Ice Age in the northeast Atlantic. - Quatern. Res. 11: 1-20.

Lamb, P.J. \& Peppler, R.A. (1987): North Atlantic Oscillation: concept and application. Bull. of the Amer. Meteorol. Soc. 60, 10: 1218-1225.

Laronne, J.B. (1990): Probability distribution of event sediment yields in the Northern Negev, Israel. - In: Boardman, J., Foster, D. L. \& Dearing, A. (eds.): Soil Erosion on Agricultural Land: 481-492; Wiley.

Le Roy Ladurie, E. (1983): Histoire du climat depuis l'An Mil. - Flammarion, Paris, 541 pp.

LESPEZ, L. (2003): Geomorphic responses to long-term land use changes in Eastern Macedonia (Greece). - Catena 51: 181-208.

Macklin, M. G., Benito, G., Gregory, K. J., Johnston, E., Lewin, J., Michczynska, D. J., Soja, R., Starkel, L. \& Thorndycraft, V.R. (2006): Past hydrological events reflected in the Holocene fluvial record of Europe. - Catena 66: 145-154.

MACKLIN, M. G., FUlLER, I. C. et al. (2002): Correlation of fluvial sequences in the Mediterranean basin over the last $200 \mathrm{ka}$ and their relationship to climate change. - Quatern. Science Revs. 21: 1633-1641.

Marston, R.A., Bravard, J.-P. \& Green, T. (2003): Impacts of reforestation and gravel mining on the Malnant River, Haute-Savoie, French Alps. - Geomorphology 55: 65-74.

Miramont, C., Jorda, C. \& Pichard, G. (1998): Evolution historique de la morphogenèse et de la dynamique fluviale d'une rivière méditerranéenne: l'exemple de la Moyenne Durance (France du SE). - Géogr. Phys. et Quatern. 52: 381-392.

Nebort-Guilhot, R. (1999): Autour du concept d'érosion accélérée: l'homme, le temps et la morphogenèse. - Géomorph., Relief, Processus, Environnem. 2: 159-172.

NOAA-NCDC-GHCN-BETA-VERSION-2 (2004): Monthly weather station precipitation data from the Global Historical Climate Network., NOAA NCDC, http://iridl.ldeo. columbia.edu/SOURCES/.NOAA/.NCDC/.GHCN/.v2beta/index.html.

OSTERKAMP, W. R. \& CostA, J. E. (1987): Changes accompanying an extraordinary flood on a sand-bed stream. - In: MAYER, L. \& NASH, D. (eds.): Catastrophic Flooding: 201-224; Allen and Unwin. 
Phillips, J. D. (2002): Geomorphic impacts of flash flooding in a forested headwater basin. J. of Hydrol. 269: 236-250.

PiCARD, M.D. \& High, L. R. (1973): Sedimentary structures of ephemeral streams. Developments in Sedimentology. - Elsevier, Amsterdam, 223 pp.

Pichard, G. (1995): Les crues sur le bas Rhône de 1.500 à nos jours. Pour une histoire hydroclimatique. - Méditerranée 3-4: 105-116.

Salvador, P. G., Bravard, J.-P., Vital, J. \& Geneva, J.-L. V. (1993): Archaeological evidence for Holocene floodplain development in the Rhône valley, France. - Z.f. Geomorphol. 88: 81-95.

Schilman, B., Bar-Mathews, M., Almogi-Labin, A. \& Luz, B. (2001): Global climate instability reflected by Eastern Mediterranean marine records during the late Holocene. Palaeogeogr., Palaeoclimatol., Palaeoecol. 176: 157-176.

Schulte, L. (2002): Climatic and human influence on river systems and glacier fluctuation in southeast Spain since the Last Glacial Maximum. - Quatern. Internat. 93-94: 85-100.

Thomas, D. S. G. (2000): Arid zone geomorphology. Process, form and change in drylands. J. Wiley, West Susex, 713 pp.

Thomson, R. \& Oldfield, F. (1986): Environmental magnetism. - Allen \& Unwin, Londres, $227 \mathrm{pp}$.

Thorndycraft, V.R. \& Benito, G. (2006): Late Holocene fluvial chronology of Spain: The role of climatic variability and human impact. - Catena 66: 34-41.

VISHER, G.S. (1969): Grain size distributions and depositional processes. - J. of Sediment. Petrol. 39, 3: 1074-1106.

VITA-FinZI, C. (1968): Late quaternary alluvial chronology of Iran. - Internat. J. of Earth Sciences 58, 2: 951-973.

- (1969): The Mediterranean Valleys. - Cambridge Univ. Press, Cambridge, 112 pp.

Woodroffe, C.D. (2002): Coasts: Form, process and evolution. - Cambridge University Press, Cambridge, $623 \mathrm{pp}$.

Xoplaki, E., Maheras, P. \& Luterbacher, J. (2001): Variability of climate in Meridional Balkans during the periods $1675-1715$ and $1780-1830$ and its impact on human life. - Climatic Change 48, 4: 581-615.

Adresses des auteurs: Dr Benoît Devillers (auteur de correspondance) CEREGE, Université de Provence, CNRS-UMR6635, Europôle de l'Arbois, 13545 Aix en Provence. Courriel: devillers@orleans.inra.fr ou bdevillers@mail.com - Dr. Nolwenn Lecuyer, LAMM, 5, rue du château de l'Horloge, BP 647, 13094 Aix-en-Provence. Courriel: Lecuyer@mmsh.univ-aix.fr. 